\title{
Study of the structural and functional state of the myocardium in women with arterial hypertension in perimenopause
}

\section{Talipova Yulduz Shavkatovna}

The Republic of Uzbekistan. Tashkent Institute for Advanced Training of Doctors. Department of Cardiology and Gerontology.

\section{Email address:}

y.talipova@mail.ru (TalipovaYulduz Shavkatovna)

To cite this article:

Talipova Yulduz Shavkatovna. Study of the structural and functional state of the myocardium in women with arterial hypertension in perimenopause. Journal of research in health science. Vol. 1, No. 2, 2018, pp. 38-44. DOI 10.26739/2523-1243

$$
\text { doi }{ }^{\circ} \text { http://dx.doi.org/10.26739/2523-1243/-2018-1-2-6 }
$$

\begin{abstract}
In present work the correlation between parameters of endothelium dysfunction within various climacterium stages features functioning of remodeling heart vessels were discovered. It is proved that correction hormonal disorders of women in perimenopause with moderate risk of cardiovascular complications, stabilization of endothelium function by selective modulators of estrogen receptors -of Klimadinon for 12 weeks in doze of $40 \mathrm{mg}$ allows to reach restoration of endothelium function and to stabilize the level of blood pressure. Efficiency of 12 weeks combined therapy klimadinon and hypotensive therapies (moxonidin) is proved, concerning achievement of target level of blood pressure, improvements of parameters recourse of remodeling hearts and vessels at women in climacterium with arterial hypertension I and II degrees.
\end{abstract}

Keywords: myocardium, hypertension, women, premenopausal, anti-hypotension treatment, cardiovascular risks.

The heart is one of the main organs the targets for cardiovascular diseases (CVD). The nature of myocardial remodeling is not monotonous and depends on many factors, the leading ones being hemodynamic and neurohumoral (Gardin, 1999, Konradi AO 2002). In addition to anatomical, features such as changes in the myocardium, in particular, diastolic dysfunction (DF) of the LV, are also a reflection of the process of its remodeling, and are now considered as one of the most important causes of heart failure [5].

At the heart of the pathogenesis of hypertension in women often is hypoestrogenism, which leads to hemodynamic disorders, mediated through increased SNS activity, changes in central mechanisms of blood 
circulation regulation, baroreceptor sensitivity, and disturbance of noradrenaline reuptake from the synaptic cleft. Increase in SNS activity is manifested by an increase in the cardiac index (SI) due to increased heart rate and increased contractile activity of the myocardium [2], changes in tone of smooth muscle cells of arteries and veins, changes in the rate of sodium reabsorption in the kidneys, and this in turn increases the venous return of blood to the heart (an increase in the tone of the veins) and resistance to blood flow (increased tonus of small arteries and arterioles). At the same time, changes in the rate of synthesis and release of presser amines are noted $[2,6]$. It is believed that the increase in BP in postmenopausal women (PostMP) is due to a significant increase in vascular resistance, as evidenced by the presence of a close positive correlation between these indicators, and the existence of a feedback between the level of progesterone and the total peripheral resistance of the vessels [5,6].

Given that the activation of the sympathetic nervous system and the presence of severe metabolic disturbances play a major role in the development of MMC, the main requirement for antihypertensive therapy should be its metabolic neutrality. These requirements are met by the preparation of the central type of action of the imidazole receptor agonist-moxonidine, which acts on the central and peripheral imidazole receptors. Moxonidine reduces the excessive activity of the sympathetic link of the autonomic nervous system, reduces adrenaline release from the adrenal medulla and norepinephrine from the ends of the sympathetic nerves, thereby exerting an antihypertensive effect, thereby increasing insulin sensitivity, leveling most of the vegetative-vascular and psychoemotional manifestations of menopausal syndrome, which allows consider it a means of pathogenetic therapy MMC. A number of studies have established the ability of moxonidine to regress LV hypertrophy, which is not inferior to the antiremodeling action of other major classes of antihypertensive drugs, and its positive effect on the systolic and diastolic function of the ventricles of the heart has also been studied [10].

In recent years, data have been obtained concerning the effect of estrogens on vascular function and structure, specific estrogen receptors have been discovered in vascular wall cells and cardiac muscle $[2,5]$. Estrogens have a direct vasodilator, the so-called endothelium-dependent property. It has been established that the use of estrogens or other alternative therapies, the appointment of an alternative to hormone replacement therapy, for example the selective modulator of estrogen receptors of plant origin Climadinone (Bionorica, Germany) in women with $\mathrm{AH}$ in menopause makes it possible to level the manifestations of estrogen diphtheria.

Purpose: the study was the study of the structural and functional state of the left ventricle of the heart in women with $\mathrm{AH}$ on the background of MMC, the evaluation of the effect of combined therapy of the selective modulator of estrogen receptors - Climadinone and imidazole receptor agonist monoxide?

Materials and methods. 101 women aged 40 to 65 years were examined. age 51.7 ë 0.6 years, of which 45 (44.6\%) cf. the age of 46.5 ë 1.3 years in premenopausal (PremM), $56(55.1 \%)$ between the ages of 56.5 ë 2.6 years in 
TalipovaYulduz Shavkatovna. Study of the structural and functional state of the myocardium in women with arterial hypertension in perimenopause.

postmenopausal women, according to the "Protocol of the study of women over 40 years of age". The main criterion for inclusion in the study was the presence of $\mathrm{AH}$.

The results of the study showed that of the total number of women surveyed, 45 (38.1\%) cf. age 46.5 ë 1.6 years were in premenopausal and $56(55.4 \%) \mathrm{cf}$. age 56.6 ë 1.5 years in postmenopausal (PostMAP). The indices of arterial pressure were determined within the limits of systolic (SBP) 140-179 $\mathrm{mm} \mathrm{Hg}$. and diastolic (DBP) of 90-109 mm Hg, which corresponds to AH I and II degree (according to the WHO / IOG classification in 2010).

Echocardiography (Echocardiography) was performed according to a standard procedure, in accordance with the recommendations of the Penn Convention Method. The following parameters of intracardiac hemodynamics were studied: end diastolic and systolic dimensions (KDR, KS, mm), end-diastolic and endsystolic and shock volumes of the LV (BWW, CSR, VO ml), their ratio to body surface area (CDI, CSI, UI), as well as the LV ejection fraction (LVEF). The mass of LV myocardium was determined (MMLV, g.), which was calculated according to the formula of R. B. Devereux et al. To correct the effect of excess body weight, the left ventricular myocardial mass index (LVMI) was determined by the formula de Simone G. et al.

The criterion of left ventricular hypertrophy in our study was LVMI $\geq 110$ $\mathrm{g} / \mathrm{m}^{2}$ (for women), the ratio of BWW / MMLD, $\mathrm{ml} / \mathrm{g}$.

According to these indicators, the following criteria for LV echo- geometry are distinguished:

- With LVMI less than $110 \mathrm{~g} / \mathrm{m}^{2}$ and
OTLC $<0.45$, the geometric model was considered normal (NG);

o With LVMI $\geq 110 \mathrm{~g} / \mathrm{m}^{2}$, RWT $<0.45$ geometry was assessed as eccentric hypertrophy of the LV (EH);

o With LVMI $\geq 110 \mathrm{~g} / \mathrm{m}^{2}$, RWT > 0.45 , concentric hypertrophy of the LV (CH) was determined;

o With LVMI <110 g / $\mathrm{m}^{2}$, RWT > 0.45 , concentric remodeling was diagnosed (A. Gunau).

The state of intracardiac hemodynamics was assessed by the parameters of blood flows on the valves mitral, aorta, tricuspid and pulmonary valve stem according to the generally accepted technique [3,5]. To assess the diastolic function of the LV, the maximum blood flow velocities were measured in the fast filling of the ventricles (PVE) and atrial systole (PVA), their ratio $(E / A)$, the percentage contribution of the systole LP to the filling of the left ventricle (AFF), the duration of the fast filling phase (PE), the acceleration time (AT), and the deceleration (DT) of the blood flow into the fast-filling phase, the duration of the atrial systolic phase (PA). In the continuous mode of Doppler echocardiography, the time of isovolytic LV relaxation (IVRT) was determined.

All studies were conducted in dynamics. The indicators were recorded at 3 observation points: initially, 1 month and 3 months after treatment.

In describing the results obtained against the background of treatment, we used the separation of patients according to the principle of ongoing treatment:

- I - group: 52 patients with AH I and II degrees belonging to the low-risk group, among them in PremMP $(n=25)$ and PostMP $(\mathrm{n}=27)$ who received Climadinone as a monotherapy ("Bionorica 
AG", Germany ), $20 \mathrm{mg}$ twice a day for 12 weeks.

- Group II: 49 patients with AH I and II degree AH belonging to the group of average risk, among them in PremMP $(\mathrm{n}=20)$ and PostMP $(\mathrm{n}=29)$, who took combination therapy - Climadinone $20 \mathrm{mg}$ twice daily and as an antihypertensive drug Physiotens ("Solvay Pharma", Germany) 0.2-0.4 mg once a day for 12 weeks.

The results were processed using various statistics methods. The reliability of differences in the comparison of arithmetic mean values was determined using the Student's test, using the "Statistica 6 for WINDOWS" software package.

Results and discussion. All women of the main group had moderate manifestations of COP. The degree of severity of CS in the Premeps differed: patients with a mild degree of $15(14.9 \%)$, with an average degree of $16(15.8 \%)$, severe - 14 (13.9\%). Likewise in PostMMP: an easy degree was found in $15(14.9 \%)$, medium - in $16(15.8 \%)$, heavy - in $25(24.8 \%)$. In $20(19.8 \%)$ of women in premenopause cf. age 49,1 \pm 1,2 years was determined by AH I degree, with the following values of the main parameters of blood pressure: average SB $147.6 \pm 3.6 \mathrm{~mm} \mathrm{Hg}$; c DAD $92.2 \pm 4.1$ $\mathrm{mm} \mathrm{Hg}$; in $22(21.8 \%)$ average age 48.2 \pm 1.9 years $\mathrm{AH}$ of II degree, with average CAD $167.2 \pm 1.96 \mathrm{~mm} \mathrm{Hg}$, in $4(3.96 \%)$ the level of SBP on the average $135,8 \pm$ 0.7 and $132 \pm 0.5 \mathrm{~mm} \mathrm{Hg}$, which corresponds to the level of a high normal blood pressure.

Among postmenopausal women, 21 $(20.8 \%)$ average age $56.3 \pm 1.7$ years were determined by grade I AG, average 147.5 $\pm 2.3 \mathrm{~mm} \mathrm{Hg}$, average, DBP $98.1 \pm 1.6$ $\mathrm{mm}$ ppm; in 30 (29.7\%) average age 57.3 \pm 1.1 years, $\mathrm{AH}$ degree II, average $\mathrm{CAD}$ $169.5 \pm 1.4 \mathrm{~mm} \mathrm{Hg}$, average DAD -101.3 $\pm 1.7 \mathrm{~mm} \mathrm{Hg}$; 3 (2.97\%) the level of SBP on the average $135.8 \pm 0.7$. Thus, in pre-menopausal hypertension, grade I and II were approximately in equal numbers, and in postmenopausal hypertension, grade II AG was 1.9 times more common than grade I AG.

During the course of treatment for 12 weeks, along with the improvement of the general condition, blood pressure significantly reached the target level: in group II, the SBP decreased to 131,50 $\pm 2,49 \mathrm{~mm} \mathrm{Hg}$. Art. $(\mathrm{p}=0.001)$, DBP $85.22 \pm 1.64 \mathrm{~mm} \mathrm{Hg}$. Art. $(\mathrm{p}=0.02)$.

I group: SBP reached the level of 119.8 $\pm 1.8 \mathrm{~mm} \mathrm{Hg}$. Art. $(\mathrm{p}=0.0001), \mathrm{DBP}-$ $80.9 \pm 1.2 \mathrm{~mm} \mathrm{Hg}$. Art. $(\mathrm{p}=0.0001)$.

Evaluation of the types of left ventricular echocardiogram at the control stage of the study showed that in the study groups, LV hypertrophy indices were determined by parameters of LV mass index (LVMI), relative wall thickness (RWT), thickness of MZV and LVL. Mean IVF in patients of this group was $0.9 \pm 0.6$, and LVMI $-125 \pm 5.3 \mathrm{~g} / \mathrm{m}^{2}$ $(\mathrm{P}<0.05)$. When assessing the types of LV geometry, the initial values were distributed as follows: normal geometry was noted in $37(36.6 \%)$; eccentric hypertrophy - in 56 (55.4\%); concentric remodeling - in 5 (4.2\%); concentric hypertrophy - in 3 (2.5\%). As pointed out by literature sources, this feature may be a consequence of more significant activation of growth factors in android obesity, which is accompanied by a more pronounced hypertrophic response. With genome obesity, hemodynamic mechanisms of hypertrophy in the form of volume overload predominate, which 
TalipovaYulduz Shavkatovna. Study of the structural and functional state of the myocardium in women with arterial hypertension in perimenopause.

creates prerequisites for the development of eccentric hypertrophy $[10,11,12]$.

The analysis of systolic function of the left ventricle revealed that the mean values of systolic function of the left ventricle, estimated from the values of PV, were distributed as follows: $\mathrm{PV}>55-$ $60 \%$ in $58(57.4 \%)$ women; $\mathrm{EF}<55 \%-$ in $34(33.7 \%)$; FV $>60 \%$ - in $9(8.9 \%)$ of the examined women. OTS $<0.45$ in 85 $(84.2 \%)$ women, OTC $>0.45$ in 16 (15.8\%) examined.

Patients in PostMP showed asymmetric hypertrophy of the LV with an increase in the relative thickness of its wall in comparison with the norm of its wall with a relative wall thickness (OTC) of $0.45 \pm 0.01$ and $0.46 \pm 0.01 \mathrm{~cm}(\mathrm{P}$ $<0.05)$ due to thickening MZHP $-1.2 \pm$ 0.02 and $1.10 \pm 0.06 \mathrm{~cm}(\mathrm{P}<0.001)$, as well as an increase in the LV mass index (IMF) of $130.5 \pm 7.3$ and $129.1 \pm 5.6 \mathrm{~g} /$ $\mathrm{m}^{2}(\mathrm{P}<0.01)$, respectively.

In the study of transmittal blood flow, a violation of the global diastolic function of the left ventricle in women with $\mathrm{AH}$ was manifested by retardation of relaxation and a violation of the diastolic filling of the LV by the "hypertrophic" type according to R. Nishimura and A. Tajik [3], which were more pronounced in group II patients. They showed an elongation in comparison with the IVRT norm in cf. $95 \pm 5.9 \mathrm{~ms}(\mathrm{P}<0.01)$, a decrease in the ratio $\mathrm{E} / \mathrm{A}-1.3 \pm 0.08$ $(\mathrm{P}<0.1)$, an increase in DT - by $30.8 \%$ $(\mathrm{P}<0.001)$. Significant disturbances in the temporal structure of LV diastole were also noted: TE was increased by $8.3 \%(\mathrm{P}<0.01)$ with a significant shortening of AT and elongation of DT (by 12.7 and $30.8 \%, P<0.001$, respectively), TA was increased by $15.4 \%$ $(\mathrm{P}<0.001)$. The results of our investigations coincide with similar studies by other authors [3].

In patients with postmenopausal women, changes in the parameters of LV diastolic function were similar to those in patients in PremEV, but disturbances in the temporal structure of diastole were more pronounced. Thus, the duration of TE and TA in patients with postmenopausal women was 11.5 and $20 \%$ higher in comparison with these indices in patients in PremMP $(0.29 \pm 0.01)$ and $(0.260 \pm 0.007) \mathrm{s}(\mathrm{P}<0,01)$. At that, the AT in patients with Premex did not differ from that in normal $(\mathrm{P}>0.05)$, and DT - was increased by $38.5 \%(\mathrm{P}<0.001)$, not differing from its value in patients with PostMP, $0.180 \pm 0.009)$ and $(0.170$ ë $0.006) \mathrm{s}(\mathrm{P}>0.05)$. The parameters of structural and functional changes in the myocardium in the examined patients are shown in the figure.

When assessing the effect of climadinone therapy on hemodynamic parameters, it was found that the systolic function of the myocardium improves statistically significantly in both the premenopausal and postmenopausal groups, with the increase in LVEF not only due to an increase in LV stroke volume, but also a decrease in its volume in systole and diastole. The degree of hypertrophy of the myocardium of the LV significantly decreases due to the (LVDM) in patients with both Prem and PostMP, but the OTC indices causing diastolic disturbances do not have dynamic changes, which is manifested by the preservation of the $\mathrm{E} / \mathrm{A}$ ratio at the level of the initial values. It was found that during the 12-week therapy, climadinone has a statistically significant effect on myocardial hypertrophy, without affecting the diastolic function. 
Against the background of combined therapy with climadinone and physiotherapy, a decrease in the degree of hypertrophy of a statistically significant character is noted, the reduction of LVDM, its indexed index (LVMI) (by 11,1 and $13,7 \%, 9,8$ and $11,4 \%$ respectively for Pre- and PostMP ); which is accompanied by an improvement in systolic and diastolic LV parameters. Moreover, in the PostMMP group, the character of changes in all parameters of cardiac hemodynamics was highly reliable, with the exception of the $\mathrm{E} / \mathrm{A}$ ratio.

From the literature it is known that in persons with excess body weight and obesity, not only an increase in the mass of the myocardium, but also a violation of diastolic function can be observed $[3,9,13]$. This circumstance is associated with excessive development of fibrosis in patients with insulin resistance [13]. Disturbances of diastolic function appear early enough and do not depend on concomitant changes in myocardial contractility, including those not disturbed. With their progression, the atrium is not fully emptied, the pressure in its cavity becomes elevated, which is clinically manifested by decompensation of the circulation. Variants of diastolic filling of the LV are not something frozen, peculiar to this patient or the type of myocardial damage. Diastolic filling of the LV can change as the disease progresses or under the influence of treatment. This is the proposed mechanism for the development of heart failure in a diastolic type, characterized by unchanged parameters of the systolic function (normal values of PV), with violation of relaxation processes due to myocardial rigidity. In this regard, it can be assumed that diastolic dysfunction at the preclinical stage is an early marker of irreversible myocardial remodeling and structural changes in the geometry of the heart.

The method of linear regression revealed a positive relationship between metabolic shifts and the morph functional state of the myocardium: TG with LVML $(p<0.05)$ and the negative correlation of the ejection fraction (EF) and SI with the level of LDL cholesterol

\section{( $p<0.05$ and $\mathrm{p}<0.01)$.}

CONCLUSIONS: Structural and functional changes in the myocardium in patients with hypertension in the per menopause were represented by asymmetric LV hypertrophy (mainly in the MZV region) with an increase in the myocardial mass and its index in the absence of dilatation of the LV cavity, which was accompanied by an increase in the end-diastolic LV wall tension and created conditions for its diastolic dysfunction, without significant violations of the systolic function.

In patients with $\mathrm{AH}$ in postmenopausal, with metabolic disorders, unidirectional changes in blood flow in the outflow tract of the LV were detected, which included preservation of the volume aortic blood flow at a normal level with a decrease in its peak velocity due to an increase in its time. Such changes in the blood flow in the aorta may reflect the presence of latent systolic LV dysfunction with unchanged rates of global contractility.

Changes in diastolic LV function in the observed patients of both groups corresponded to the hypertrophic type of diastolic dysfunction. They were caused as a violation of active relaxation of the myocardium. At the same time, disturbances in volume and velocity 
TalipovaYulduz Shavkatovna. Study of the structural and functional state of the myocardium in women with arterial hypertension in perimenopause.

parameters of LV diastolic filling in both groups were equally expressed, then the temporal structure of diastole and more severe diastolic LV dysfunction were observed in patients in PostMP.

The inclusion of moxanidine and climadinone in the treatment complex of patients with $\mathrm{AH}$ on the background of MMC promoted the achievement of blood pressure to the target values and optimized the indices of diastolic filling of the LV. The normalizing effect of such therapy on the parameters of intracardiac hemodynamics in the examined patients with MMC is probably due to both a decrease in the stiffness of the left ventricle chamber and an increase in diastole due to a decrease in heart rate.

\section{References}

1. Anichkov DA, Shostak NA, Kopytova AA Arterial hypertension in women with metabolic syndrome: the role of general and abdominal obesity. // Clinician No. 4 2006. P. 21-25

2. Bart B.Ya., Boronenkov GM, Benevskaya V.F. Arterial hypertension in postmenopausal women: modern possibilities of drug therapy in polyclinic conditions. Russian Cardiology Journal 2001. №5 with 69-70.

3. Konradi AO, Zhukova AV, Vinnik TA Structural and functional parameters of the myocardium in patients with hypertensive disease, depending on body weight, type of obesity and the state of carbohydrate metabolism.

4. Nekrutenko LA, Agafonov AV, Lykova DA Endothelial dysfunction is a risk factor for hypertension in the elderly. Abstracts of the reports of the Russian National Congress of Cardiology "Cardiology: Efficiency and Safety of Diagnosis and Treatment" October 2001.

5. Smetnik VP, Shestakova IG Menopause and cardiovascular system. Therapeutic archive of 1999. 71. №10 with 61-65.

6. Shekstakova MV Endothelial dysfunction - the cause or effect of the metabolic syndrome? Rus. honey. journal. 2001; 9 (2).

7. Deanfield J, Donald A, Ferri C, Giannattasio C et al. Endothelial function and dysfunction. Part I: Methodological issues in different vascular beds: A statement by the Working Group on the Endothelin and Endothelial Factors of the European Society of Hypertension. J Hypertes 2005 Jan; 23 (1): 7-17. 8. Celermajer DS. Endothelial dysfunction: does it matter? Is it reversible? J Am Coll Cardiol 1997; 30 (3): $325-33$.

9. Canau A., Devereux R. B., Roman M.J. et al. Patterns of left ventricular hypertrophy and geometric remodeling in essential hypertension / J. Amer.Coll.Cardiol.1992; 19: 1550-1558

10. Nishimura R.A., Tajik A.J. Quantitative hemodynamics by Doppler echocardiography: A noninvasive alternative to cardiac catheterization // Prog. Cardiovasc. Dis. - 1994. - Vol. 36.-P. 309-342.

11. Spring A., Haczynski J., Przewlocka-Kosmala M. et al. Effect of moxonidine on left ventricular hypertrophy in hypertensive patients // J. Clin. Basic Cardiology. - 2001. - No. 4. - P. 1-5.

12. Sundstrum $\mathbf{J}$, Lind $\mathrm{L}$, Nystrum $\mathbf{N}$ et al.:eft ventricular concentric remodeling ather than left ventricular hypertrophy is related to insulin resistance syndrome in the elderly. Circulation 2000; 101: 2595-600.

13. Thakur V, Richards R, Reisin E. Obesity, hypertension, and the heart. Am J Med Sci 2001; 321 (4): $242-8$. 